\title{
Variabilité génétique de la ponctuation noire sur la truitelle Fario (Salmo trutta L.)
}

\author{
J.M. BLANC, H. POISSON et R. VIBERT \\ Laboratoire d'Ecologie des Poissons et d'Aménagement des Pêches, \\ Centre de Recherches hydrobiologiques, Saint-Pée-sur-Nivelle \\ F 64310 Ascain
}

\begin{abstract}
Résumé
Le nombre de points noirs de la robe a été déterminé, chez les truitelles de 25 familles de truite fario, séparément de chaque côté du corps et dans quatre zones : operculaire, dorso-antérieure, dorso-postérieure et ventrale. L'analyse de ces mesures met en évidence :

- l'absence de dissymétrie gauche droite collective;

- une relation de proportionnalité entre la ponctuation et la longueur corporelle ;

- indépendamment des variations de taille, une variabilité génétique concernant la ponctuation dorsale (héritabilité estimée : 0,4);

- l'existence d'une variation relativement peu importante de la répartition antéropostérieure et dorso-ventrale de la ponctuation.
\end{abstract}

Ces résultats sont discutés dans le contexte de la discrimination entre populations d'origine sauvage ou piscicole.

\section{Introduction}

Dans un article récent, Chaumont (1980) signale que les saumons (Salmo salar L.) du nord du Québec (rivière George) ont des ponctuations noires beaucoup plus nombreuses que ceux du sud de la province (rivière Matane). Il considère que les différences observées devraient attirer l'attention des généticiens et que c'est à eux de déterminer si cette pigmentation est suffisamment héritable pour constituer un critère d'identification génétique.

Effectivement, un caractère de robe tel que la ponctuation noire, bien qu'a priori dénué d'intérêt économique intrinsèque, peut être utile comme marqueur génétique chez les Salmonidés, soit qu'il constitue naturellement un critère de discrimination entre certaines populations sauvages, soit qu'il puisse faire l'objet de sélections artificielles dans des souches piscicoles justifiant un tel marquage.

Cet intérêt pratique potentiel ne semble toutefois pas avoir motivé les chercheurs : un examen de la littérature existante relative à la ponctuation de la robe chez les Salmonidés montre que la part génétique de la variabilité de ce caractère n'a jamais 
été rigoureusement estimée, les échantillons observés étant généralement prélevés dans les milieux d'origine des populations étudiées. Des résultats probants ont néanmoins été obtenus par QADRI (1959) sur la truite à gorge coupée (Salmo clarkii Richardson) et par TACK (1973) sur la truite arc-en-ciel (Salmo gairdneri Richardson).

Cette situation a incité les auteurs à utiliser un stock disponible adéquat de truite fario, Salmo trutta L., pour faire une première évaluation de l'importance relative des facteurs génétiques et environnementaux agissant sur la ponctuation noire de la robe de cette espèce, intra-souche et en milieu piscicole.

\section{Matériel et méthodes}

\section{Matériel expérimental et mesures}

L'étude a porté sur 250 truitelles de 5 à $10 \mathrm{~cm}$ conservées dans une solution de formol à 10 p. 100, à l'obscurité, depuis trois ans. D'après IsLAM et al. (1973), ce mode de conservation n'apporte aucune altération au comptage de la ponctuation noire chez la truite arc-en-ciel, espèce voisine de la fario.

Ces truitelles étaient issues d'un élevage d'évaluation sur descendance de 25 mâles (croisés avec un ensemble de femelles) réalisé à la pisciculture de Saint-Pée-sur-Nivelle (Pyrénées-Atlantiques) à l'aide de reproducteurs (mâles de 2 ans, femelles de 3 ans) choisis au hasard dans la souche de la pisciculture. Elles avaient été élevées dans un ensemble d'auges aménagées en 50 compartiments identiques, chaque famille de demi-frères-sœurs étant répliquée dans deux compartiments choisis au hasard. A l'âge de 7 mois, 5 truitelles prises au hasard dans chaque compartiment (soit 10 par famille) avaient été sacrifiées et formolées en vue d'études anatomiques.

Le nombre de points noirs a été déterminé de chaque côté du corps et dans quatre zones latérales (fig. 1) :

- zone operculaire (opercule et joue) ;

- zone dorso-antérieure (au-dessus de la ligne latérale et en avant de l'aplomb antérieur de la nageoire dorsale) ;

- zone dorso-postérieure (au-dessus de la ligne latérale et en arrière de l'aplomb antérieur de la nageoire dorsale);

- zone ventrale (en dessous de la ligne latérale).

En outre, la longueur corporelle de chaque sujet a été mesurée.

\section{Méthodes statistiques}

Le modèle d'analyse de variance utilisé est hiérarchique à effets aléatoires (FEDERER, 1955). $X_{\mathrm{ijk} 1}$ étant la mesure réalisée (dans une zone latérale ou dans un ensemble de zones) du côté gauche $(1=1)$ ou droit $(l=2)$ sur le $\mathbf{k}^{\mathbf{e}}$ poisson $\mathrm{du} \mathrm{j}^{\mathrm{e}}$ replicat attribué à la ie famille,

$$
X_{i j k I}=\mu+F_{i}+R_{i j}+P_{i j k}+E_{i j k I}
$$




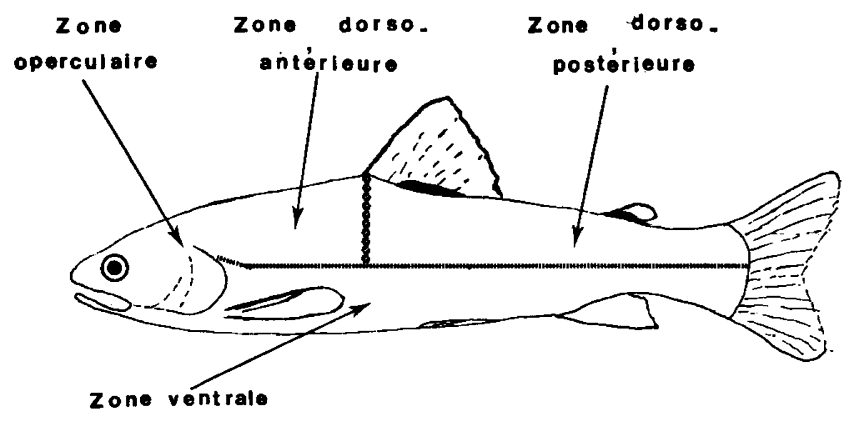

FIG. 1

Zones de comptage de la ponctuation noire.

Areas where black spots were counted.

- $\mu$ est l'espérance du caractère considéré,

- $F_{i}, R_{i j}$ et $P_{i j k}$ sont respectivement l'effet paternel (famille), environnemental de groupe (replicat intra-famille) et individuel (poisson intra replicat),

- $\mathrm{E}_{\mathrm{ijk} \mathrm{l}}$ est l'erreur résiduelle (gauche - droite).

Les méthodes d'estimation des composantes de variance et de covariance ainsi que l'interprétation génétique sont tirées de BECKER (1967), la variance de l'effet paternel représentant le quart de la variance génétique additive (FALCONER, 1960).

Enfin, la méthode d'analyse en composantes principales (LEFEBVRE, 1980) a été utilisée pour décrire la variation conjointe de la ponctuation dans les zones corporelles étudiées.

\section{Résultats}

Les histogrammes et paramètres de distribution des 500 comptages réalisés dans chaque zone (fig. 2) montrent l'importante variabilité phénotypique de la ponctuation, exception faite de la zone operculaire, ainsi qu'une légère asymétrie commune à toutes les distributions.

L'analyse de variance relative aux effets latéraux (gauche-droite) sur la ponctuation met en évidence l'absence d'effet latéral significatif, ce qui justifie le modèle utilisé prenant en compte l'erreur latérale comme source de variation résiduelle uniquement.

\section{Analyse de la variation entre truitelles}

L'analyse de variance effectuée d'après ce modèle hiérarchique sur chacune des quatre zones (tabl. 1) met en évidence :

- un effet familial faiblement significatif sur les zones dorsales; 

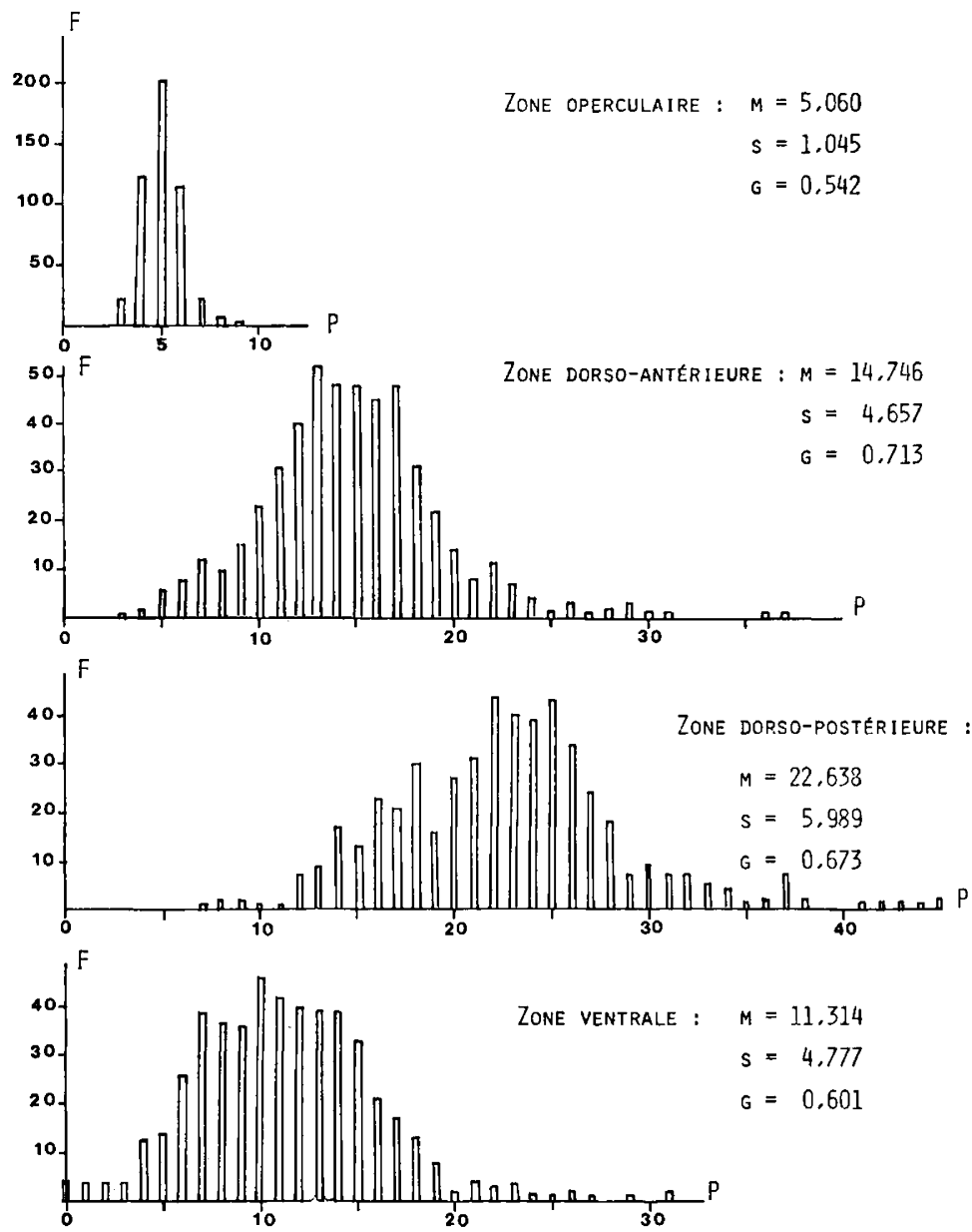

Fig. 2

Distribution des mesures de ponctuation. Distribution of spot counts.

$P$ : nombre de points noirs.

F : fréquence absolue (nombre de mesures).

M : moyenne.

S : écart-type.

G : coefficient d'asymétrie. ventrales ;

un effet environnemental (replicat) significatif sur les zones operculaires et

- et une grande variabilité inter-individuelle à l'intérieur des compartiments d'élevage, d'autant plus significative que la variation résiduelle (erreur latérale) est minime. 


\section{Relation entre la ponctuation et la longueur corporelle}

Les carrés moyens et produits moyens relatifs à la ponctuation totale des quatre zones $(\mathrm{X})$ et à la longueur corporelle $(\mathrm{L}$, en $\mathrm{mm}$ ) sont également présentés dans le tableau 1. La corrélation phénotypique entre les deux caractères est d'environ 0,7 (valeur hautement significative) et est essentiellement liée à la variation entre individus intra-replicats. Le nuage de points correspondant est représenté sur la figure 3.

Bien que ces données puissent être ajustées à un modèle linéaire simple, un calcul des écarts à la régression et des résidus latéraux montre que ceux-ci augmentent sensiblement avec la longueur des sujets, et cette constatation a conduit à préférer l'hypothèse d'une relation de proportionnalité. Pour vérifier l'isométrie de cette relation, il a été procédé à un ajustement à une fonction puissance : $X=\alpha L^{\beta}$ (SoKAL $\&$ RoHLF, 1969). Les estimations obtenues pour l'exposant entre familles $(\beta=1,17$ d'écart-type 0,37$)$, entre replicats intra-familles $(\beta=1,25$ d'écart-type 0,43$)$ et entre individus intra-replicats $(\beta=1,07$ d'écart-type 0,08$)$ ne diffèrent pas significativement entre elles, ni par rapport à l'unité.

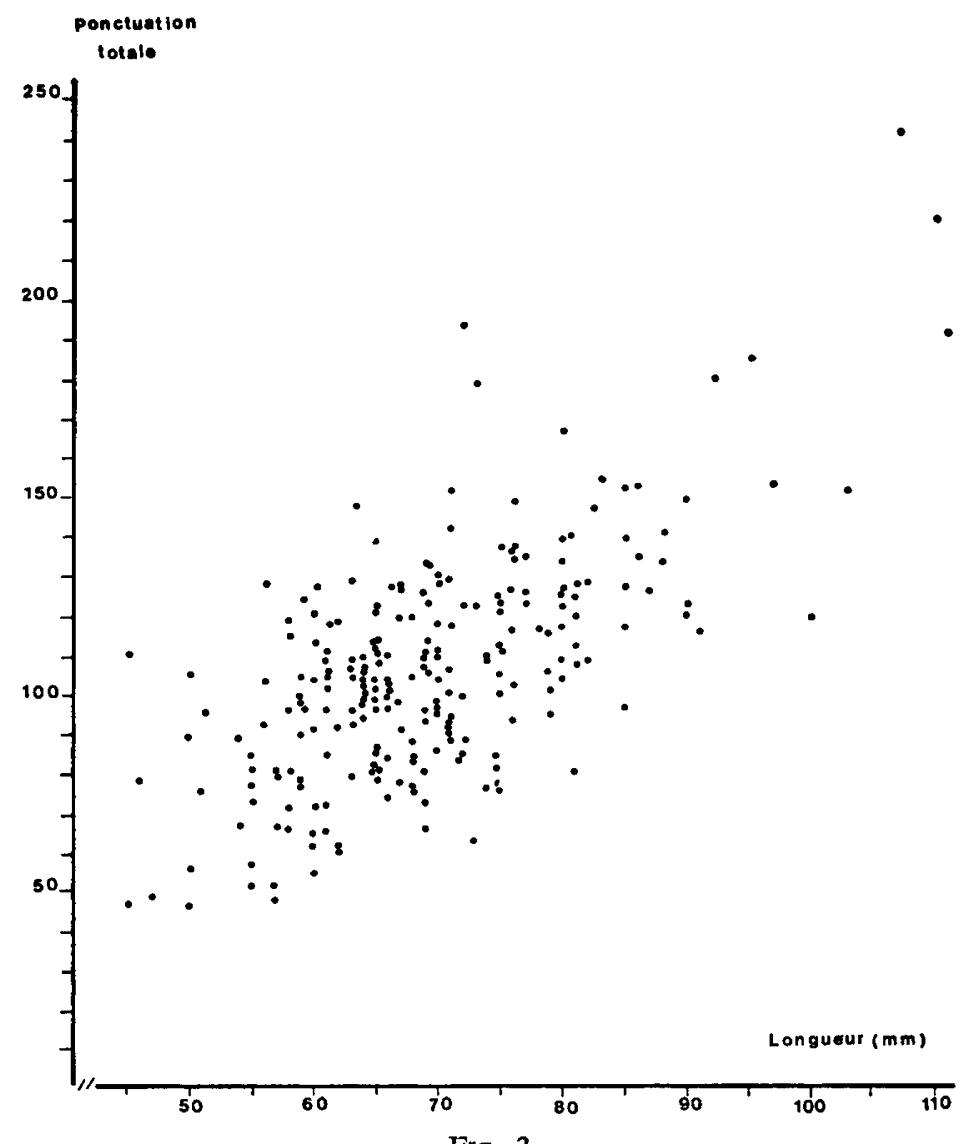

Fig. 3

Relation entre la ponctuation totale (bilatérale) et la longueur corporelle. Relationship between total spotting (both sides) and body length. 


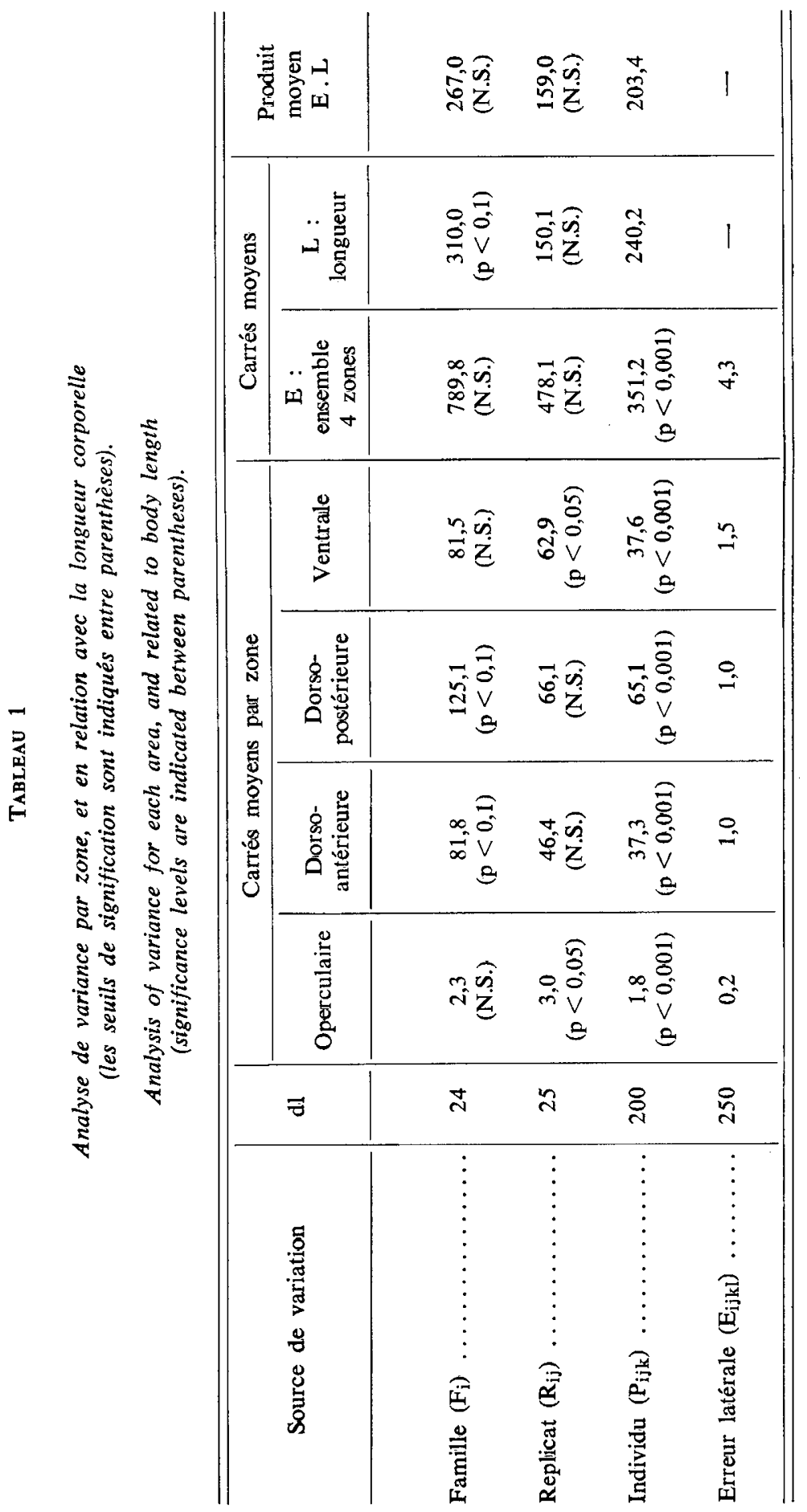


Compte tenu de ces résultats, la poursuite de l'analyse a été effectuée sur la ponctuation relative $\mathrm{Y}=100 \frac{\mathrm{X}}{\mathrm{L}}$ correspondant à une longueur corporelle de $100 \mathrm{~mm}$, soit 10 centimètres.

\section{Décomposition de la variance de la ponctuation relative}

L'analyse de variance effectuée sur la ponctuation relative rend plus perceptible l'effet familial sur les zones dorsales, qui devient significatif au seuil de 0,05. Les composantes de variance calculées d'après cette analyse sont présentées dans le tableau 2. La variance génétique additive, estimée par le quadruple de $\sigma_{\mathrm{F}}^{2}$, représente environ 40 à 50 p. 100 de la variance totale dans les zones dorsales. La variance due à l'environnement de groupe est mesurée par $\sigma_{\mathrm{R}}^{2}$ et n'apparaît nettement que dans la zone ventrale. La composante $\sigma_{\mathrm{P}}^{2}$ enfin constitue environ les trois quarts de la variance totale dans toutes les zones. Même en tenant compte du fait que cette composante entre demi-frères-sœurs contient les trois quarts de la variance génétique additive (BECKER, 1967), il reste une variation importante explicable par des effets génétiques non additifs, des effets maternels ou des effets environnementaux individuels.

\section{TABLeAU 2}

Composantes de la variance de la ponctuation corrigée $Y=100 \mathrm{X} / \mathrm{L}$ (les écarts-types d'erreur approximatifs sont indiqués entre parenthèses, et les composantes significatives à $p<0,05$ sont soulignées).

Variance components of adjusted spotting $Y=100 \mathrm{X} / \mathrm{L}$ (approximate standard errors are indicated between parentheses, and components which are significant at 0.05 level are underlined).

\begin{tabular}{|c|c|c|c|c|}
\hline Source de variation & Z. operculaire & $\begin{array}{l}\text { Z. dorso- } \\
\text { antérieure }\end{array}$ & $\begin{array}{l}Z \text { Z. dorso- } \\
\text { postérieure }\end{array}$ & $Z$. ventrale \\
\hline \multirow[t]{2}{*}{ Famille $: \sigma_{\mathrm{F}}^{2} \ldots \ldots \ldots$} & $-0,01$ & 3,01 & 4,88 & 0,97 \\
\hline & $(0,12)$ & $(1,92)$ & $(2,81)$ & $(2,62)$ \\
\hline \multirow{2}{*}{ Replicat $: \sigma_{\mathrm{R}}^{2} \ldots \ldots \ldots \ldots$} & 0,22 & 1,51 & 1,70 & 6,03 \\
\hline & $(0,17)$ & $(1,79)$ & $(2,46)$ & $(3,45)$ \\
\hline \multirow{2}{*}{ Individu $: \sigma_{\mathrm{P}}^{2} \ldots \ldots \ldots \ldots$} & 1,83 & 23,10 & 33,62 & 30,65 \\
\hline & $(0,20)$ & $(2,41)$ & $(3,46)$ & $(3,20)$ \\
\hline Erreur $: \sigma_{\mathrm{E}}^{2}$ & $\begin{array}{r}0,39 \\
(0,03) \\
\end{array}$ & $\begin{array}{c}2,18 \\
(0,19) \\
\end{array}$ & $\begin{array}{c}2,33 \\
(0,21)\end{array}$ & $\begin{array}{c}3,01 \\
(0,27)\end{array}$ \\
\hline Total $\quad: \sigma_{\mathrm{Y}}^{2} \ldots \ldots \ldots$ & 2,43 & 29,80 & 42,53 & 40,66 \\
\hline Héritabilité $: h^{2}=\frac{4 \sigma_{\mathrm{F}}^{2}}{\sigma_{\mathrm{Y}}^{2}} \ldots$ & $\begin{array}{r}-0,02 \\
(0,20)\end{array}$ & $\begin{array}{c}0,40 \\
(0,26)\end{array}$ & $\begin{array}{c}0,46 \\
(0,26)\end{array}$ & $\begin{array}{c}0,10 \\
(0,26)\end{array}$ \\
\hline
\end{tabular}




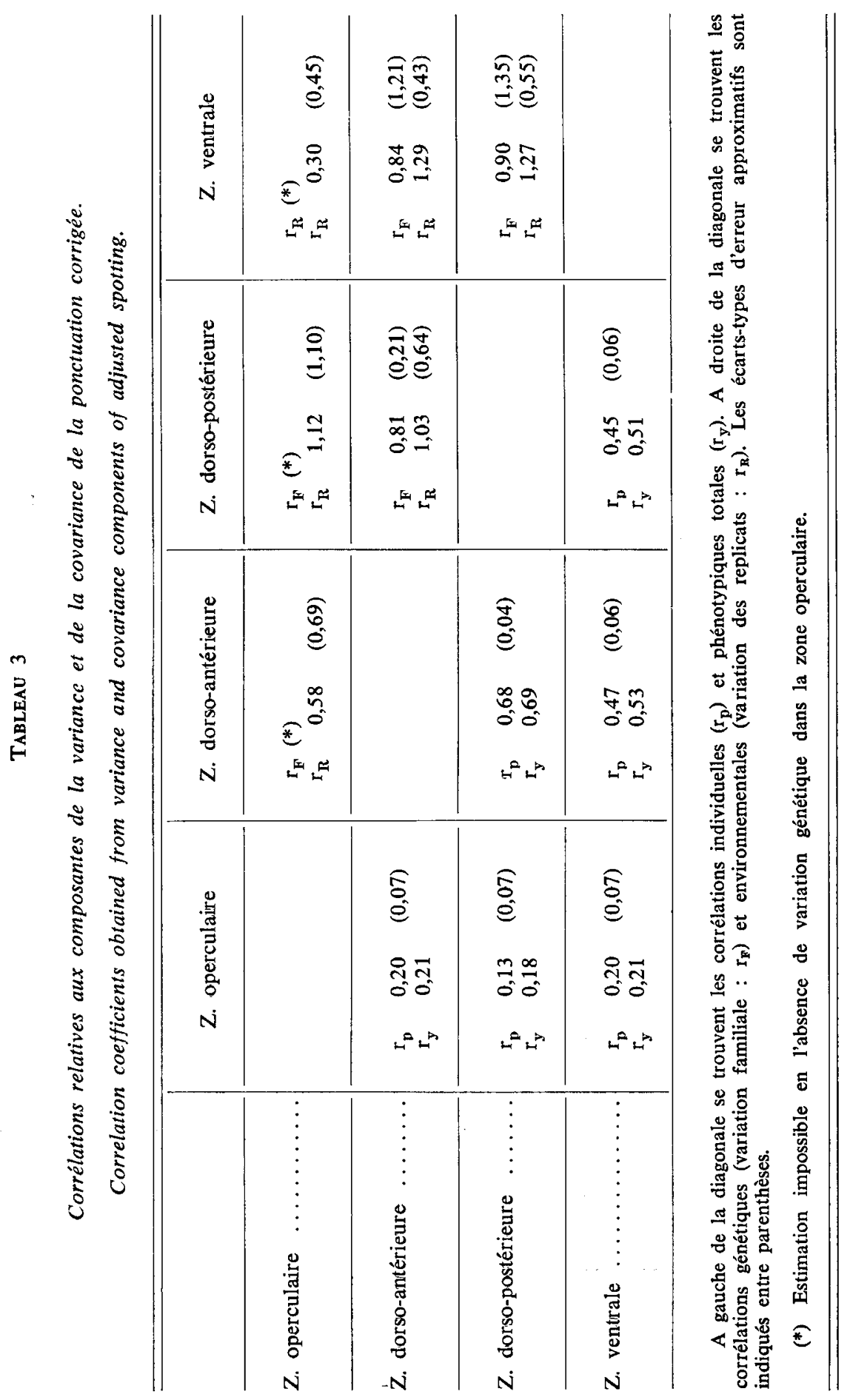




\section{Corrélations entre zones et composantes principales de la variation}

Les corrélations entre zones dues aux composantes des variances et covariances des effets famille, replicat, individu, totaux, ont été estimées (tabl. 3). Compte tenu des erreurs d'estimations, la seule corrélation génétique significative est celle concernant les deux zones dorsales; l'existence de corrélations environnementales positives entre les zones dorso-antérieure, dorso-postérieure et ventrale paraît hautement probable sans qu'on puisse les chiffrer avec précision; enfin, les corrélations d'origine individuelle intra replicats, de loin les plus précises, montrent la relative indépendance entre la ponctuation operculaire et celle des autres zones.

Pour mieux décrire la variation spatiale de la ponctuation sur le corps, des analyses en composantes principales ont été effectuées sur les matrices des composantes de variances et de covariances génétiques additives $\left(4 \sigma_{\mathrm{F}}^{2}\right)$ et individuelles non génétiques additives $\left(\sigma_{\mathrm{P}}^{2}-3 \sigma_{\mathrm{F}}^{2}\right)$, en excluant la zone operculaire pour laquelle l'estimée de $\sigma_{F}^{2}$ est négative. Les résultats obtenus figurent dans le tableau 4. Les vecteurs propres (combinaison linéaire des trois ponctuations zonales, de variance maximum et de covariances nulles) calculés dans les deux analyses sont analogues mais rendent compte de proportions différentes des variations étudiées. La composante la plus importante dans les deux cas et surtout pour la variation génétique additive, correspond à un facteur général de ponctuation dans les trois zones. La seconde composante de la variation individuelle non génétique traduit les tendances dorso-ventrales de la ponctuation; elle ne fait par contre l'objet que d'une part négligeable de variation génétique additive. Une composante enfin rend compte des tendances antéro-postérieures en opposant presque exclusivement les deux zones dorsales.

TABLEAU 4

Analyses en composantes principales (zone operculaire exclue).

Principal component analyses (opercular area excluded).

\begin{tabular}{|c|c|c|c|c|c|c|}
\hline \multirow{2}{*}{$\begin{array}{r}\text { Composante } \mathrm{n}^{\circ} \text { (par ordre } \\
\text { d'importance) } \\
\ldots\end{array}$} & \multicolumn{3}{|c|}{$\begin{array}{l}\text { a) D'après les composantes } \\
\text { génétiques additives }\end{array}$} & \multicolumn{3}{|c|}{$\begin{array}{l}\text { b) D'après les composantes } \\
\text { individuelles } \\
\text { non génétiques additives }\end{array}$} \\
\hline & 1 & 2 & 3 & 1 & 2 & 3 \\
\hline Valeur propre $\ldots \ldots \ldots$ & 32,15 & 2,76 & 0,54 & 41,50 & 16,30 & 6,74 \\
\hline $\begin{array}{l}\text { \% variance analysée } . . . . \\
\text { Coefficients des vecteurs } \\
\text { propres : }\end{array}$ & 90,7 & 7,8 & 1,5 & 64,3 & 25,3 & 10,4 \\
\hline $\begin{array}{l}\text { Dorso-antérieur } \ldots \ldots \ldots \\
\text { Dorso-postérieur } \ldots \ldots \ldots \\
\text { Ventral } \ldots \ldots \ldots \\
\end{array}$ & $\begin{array}{l}0,56 \\
0,76 \\
0,33\end{array}$ & $\begin{array}{r}-0,81 \\
0,59 \\
0,03\end{array}$ & $\begin{array}{r}-0,16 \\
-0,28 \\
0,94\end{array}$ & $\begin{array}{l}0,45 \\
0,53 \\
0,72\end{array}$ & $\begin{array}{r}-0,37 \\
-0,62 \\
0,69\end{array}$ & $\begin{array}{r}-0,81 \\
0,58 \\
0,08\end{array}$ \\
\hline
\end{tabular}


A titre de vérification, une analyse semblable a été effectuée à partir des variances et covariances phénotypiques totales, zone operculaire y comprise : les trois premières composantes sont analogues à celles obtenues au tableau $4 \mathrm{~b}$ et ne font pratiquement pas intervenir la pigmentation operculaire; celle-ci se reporte dans la quatrième et dernière composante, responsable de moins de 2 p. 100 de la variation totale.

\section{Discussion et conclusion}

Entreprise pour apporter des indications préliminaires sur l'hérédité des caractères de robe chez les salmonidés, cette étude présente certaines insuffisances qui en limitent la portée :

- La taille de l'échantillon étudié, trop faible pour une analyse génétique précise, est à l'origine d'erreurs d'estimation notables.

- Les mesures ont été faites sur des truitelles dont la ponctuation, liée à la taille corporelle, n'est donc pas immuable. IsLAM et al. (1973) ont observé la même relation chez des truites arc-en-ciel de 10 à $30 \mathrm{~cm}$ de long, et ont en outre constaté que la ponctuation devenait indépendante de la taille chez les individus de plus de $30 \mathrm{~cm}$.

- La population étudiée provenait d'une souche de pisciculture ayant une base génétique assez large et n'ayant jamais fait l'objet d'une sélection dirigée. Etant donnée toutefois la tendance naturelle des pisciculteurs à choisir, au moins parmi les mâles, de «jolis » sujets pour la reproduction, on ne peut être absolument sûr que la souche ait conservé la totalité de sa variabilité génétique pour le caractère de robe considéré.

- Il reste enfin que cette étude, réalisée intra-souche et dans des conditions de milieu particulièrement homogènes, ne peut donner aucune certitude quant aux différences génétiques et environnementales pouvant exister entre des populations naturelles ou piscicoles d'origines différentes.

Ces réserves étant faites, un certain nombre de résultats paraissent mériter considération en vue des études ultérieures :

- L'absence de dissymétrie collective gauche-droite significative, ainsi que la faible importance de l'erreur latérale résiduelle permettent de limiter les comptages à un seul côté sur chaque individu.

- Compte tenu de sa faible variabilité, la ponctuation operculaire apparaît n'avoir qu'un intérêt secondaire, sauf différences possibles entre populations.

- La ponctuation ventrale, considérée isolément ou par opposition avec la ponctuation dorsale, se révèle très sensible aux variations environnementales malgré l'homogénéité apparente des conditions d'expérience.

- Seule la ponctuation dorsale présente une variabilité génétique additive notable, propice à la sélection. Les neuf dixièmes de cette variabilité apparaissent liés à un facteur général de ponctuation. La fraction restante, relative à la localisation antéro-postérieure de cette ponctuation, n'est toutefois pas dénuée d'intérêt, compte tenu de l'impact relativement faible des effets environnementaux à ce niveau.

Ce dernier résultat est à rapprocher de celui de QADRI (1959) qui a pu discriminer nettement deux sous-espèces de truite à gorge coupée, Salmo clarkii clarkii et S.c. lewisi, 
par la localisation antéro-postérieure de la ponctuation. Il est à noter par ailleurs que entre ces deux sous-espèces, les ponctuations des zones operculaires et ventrales sont beaucoup plus discriminantes que dans la présente étude.

En outre, chez la truite arc-en-ciel, Salmo gairdneri, TACK (1973) a montré que les individus issus d'un couple de reproducteurs à ponctuation ventrale élevée avaient eux-mêmes une ponctuation ventrale supérieure à la moyenne de la population, l'héritabilité ainsi réalisée étant de l'ordre de 0,3 .

En tout état de cause, il n'apparaît pas facile d'envisager le marquage génétique de souches en pisciculture par la ponctuation noire, la variation non sélectionnable restant une source de confusions fâcheuses.

Vis-à-vis du milieu naturel par contre, les recherches relatives à ce caractère mériteraient d'être poursuivies en vue d'une meilleure discrimination des populations sauvages. Egalement, moyennant l'acceptation d'un certain risque d'erreur, on pourrait utiliser des souches sélectionnées à cet effet sur un critère de ponctuation pour tester expérimentalement sans l'artefact du marquage l'efficacité des méthodes de repeuplement dans des milieux déjà occupés par une population locale.

Reçu pour publication le 31 mars 1982.

\section{Summary}

Genetic variability of black punctuation on brown trout (Salmo trutta L.)

The number of black spots on the body surface was determined, among fingerlings from 25 families of brown trout, on each side of the body and in four areas : opercular, dorsoanterior, dorso-posterior and ventral. Analysis of these data brings to evidence :

- the lack of lateral asymmetry of collective origin;

- a proportional relationship between spotting and body length;

- a size-free genetic variability pertaining to the dorsal spotting (estimated heritability : 0.4 );

- the existence of a relatively weak variation of the anterio-posterior and dorso-ventral location of the spots.

These results are discussed in reference to the discrimination between wild as well as hatchery bred populations.

\section{Références bibliographiques}

BECKER W.A., 1967. Manual of procedures in quantitative genetics. Wash. State Univ. Press (2nd ed.), Pullman, Was., 130 p.

Chaumont G., 1980. La pigmentométrie. Une méthode d'identification génétique...? Saumons, 32, 10.

FALCONER D.S., 1960. Introduction to quantitative genetics. Oliver and Boyd Ed., Edinburgh, $365 \mathrm{p}$. 
FEDerer W.T., 1955. Experimental design. Theory and application. The MacMillan Company, New York, 544 p.

ISLAM M.A., Nose Y., YASUdA F., 1973. Number and distribution pattern of the black spots on body surface of rainbow trout. Bull. Jap. Soc. sci. Fish., 39, 727-739.

LEFEBVRE J., 1980. Introduction aux analyses statistiques multidimensionnelles. Masson $\left(2^{\circ}\right.$ éd.), Paris, 259 p.

QADRI S.U., 1959. Some morphological differences between the subspecies of cutthroat trout, Salmo clarkii clarkii and Salmo clarkii lewisi in British Columbia. J. Fish. Res. Bd Can., 16, 903-922.

Sokal R.R., Rohlf F.J., 1969. Biometry. Freeman, San Francisco, Cal., 776 p.

TACK E., 1973. Züchtungsfragen in der Forellenzucht. Osterreichs Fischerei, 26, 77-84. 